Effective Hamiltonian approach to the non-Markovian dynamics in a spin bath

This article has been downloaded from IOPscience. Please scroll down to see the full text article.

2010 Phys. Scr. 2010014021

(http://iopscience.iop.org/1402-4896/2010/T140/014021)

View the table of contents for this issue, or go to the journal homepage for more

Download details:

IP Address: 147.163.21.159

The article was downloaded on 10/01/2011 at $14: 13$

Please note that terms and conditions apply. 


\title{
Effective Hamiltonian approach to the non-Markovian dynamics in a spin bath
}

\author{
E Ferraro ${ }^{1}$, H-P Breuer ${ }^{2}$, A Napoli ${ }^{1}$ and A Messina ${ }^{1}$ \\ ${ }^{1}$ CNISM and Dipartimento di Scienze Fisiche ed Astronomiche, Università di Palermo, via Archirafi 36, \\ 90123 Palermo, Italy \\ ${ }^{2}$ Physikalisches Institut, Universität Freiburg, Hermann-Herder-Strasse 3, D-79104 Freiburg, Germany \\ E-mail: ferraro@fisica.unipa.it and breuer@physik.uni-freiburg.de
}

Received 29 March 2010

Accepted for publication 16 June 2010

Published 30 September 2010

Online at stacks.iop.org/PhysScr/T140/014021

\begin{abstract}
We investigate the dynamics of a central spin that is coupled to a bath of spins through a non-uniform distribution of coupling constants. Simple analytical arguments based on master equation techniques as well as numerical simulations of the full von Neumann equation of the total system show that the short-time damping and decoherence behaviour of the central spin can be modelled accurately through an effective Hamiltonian involving a single effective coupling constant. The reduced short-time dynamics of the central spin is thus reproduced by an analytically solvable effective Hamiltonian model.
\end{abstract}

PACS numbers: 03.65.Yz, 42.50.Lc, 03.65.Ta, 73.21.La

(Some figures in this article are in colour only in the electronic version.)

\section{Introduction}

Recent years have seen a growing interest in spin systems, particularly in connection with serious efforts in attempting to build scalable quantum computers [1-6]. Electron spins in semiconductor structures are indeed ideal candidates to realize the basic element in quantum information processing, namely a qubit [7]. In this sense it is extremely important to understand qualitatively and quantitatively all aspects of electron spin decoherence in solids that represent the main obstacle in quantum computing and information processing. A serious possible limitation of spin coherence in semiconductors is the hyperfine interaction with the surrounding nuclear spins [8]. It can lead to spin decoherence either through direct electron-nuclear spin flips or through an effective intra-bath interaction.

In this paper we consider a system composed of a single electron spin non-uniformly coupled to a bath of $N$ nuclear spins that is modelled by the Hamiltonian

$$
H=\frac{\omega_{0}}{2} \sigma_{3}+\sum_{k=1}^{N} \frac{\omega}{2} \sigma_{3}^{k}+\sum_{k=1}^{N} \alpha_{k} \vec{\sigma} \cdot \vec{\sigma}^{k},
$$

where the Pauli operators $\vec{\sigma}$ and $\vec{\sigma}^{k}$ act on the Hilbert space of the central spin and of the $k$ th bath spin, respectively.
The coefficients $\alpha_{k}, k=1, \ldots, N$, take into account the strength of the spin-bath coupling and $\omega_{0}(\omega)$ quantifies the Zeeman splitting due to an external magnetic field acting on the central spin (bath spins). The interest towards the model (1), witnessed by the numerous papers that appeared in the literature during the last few years [9-12], undoubtedly stems from its versatility in describing very different systems in various physical scenarios with many applications. In the case of a non-uniform distribution of the coupling constants $\alpha_{k}$, the analysis of the physical properties of the system is however extremely difficult and several efficient numerical simulation techniques [13, 14] and analytical methods based on non-Markovian master equation and projection operator techniques [15-17] have been developed.

The aim of this paper is to develop a rather different strategy, namely to explore the possibility of reproducing the dynamical behaviour, resulting from the Hamiltonian (1) with a non-uniform distribution for the couplings, through the use of an effective Hamiltonian involving a single suitably chosen effective coupling $\alpha_{\text {eff }}$. The question is thus whether it is possible to reproduce the short-time dynamics of the populations and coherences of the central spin by means of an effective Hamiltonian model. In particular, the effective Hamiltonian approach should be able to model correctly the short-time damping and decoherence behaviour of the 
central spin. The great advantage of such an approach is obviously given by the fact that the model corresponding to the Hamiltonian (10) with uniform couplings is exactly solvable analytically.

\section{Time-local master equation}

Hamiltonian (1) can be written as $H=H_{0}+H_{\mathrm{I}}$, where

$$
H_{0}=\frac{\omega_{0}}{2} \sigma_{3}+\sum_{k=1}^{N} \frac{\omega}{2} \sigma_{3}^{k}+2 \sigma_{3} K_{3}
$$

represents the unperturbed part, and

$$
H_{\mathrm{I}}=2\left(\sigma_{+} K_{-}+\sigma_{-} K_{+}\right)
$$

is the interaction Hamiltonian [2]. $\sigma_{ \pm}$are the raising and lowering operators of the central spin and

$$
\begin{aligned}
& K_{3}=\frac{1}{2} \sum_{k=1}^{N} \alpha_{k} \sigma_{3}^{k}, \\
& K_{ \pm}=\sum_{k=1}^{N} \alpha_{k} \sigma_{ \pm}^{k} .
\end{aligned}
$$

In the following we work in the interaction picture with respect to $H_{0}$ and denote by $\Pi_{m}$ the bath projection operator that projects onto the eigenspace of the three-component of the total spin of the bath belonging to the eigenvalue $m=-\frac{N}{2}, \cdots,+\frac{N}{2}$. The density matrix $\rho_{\mathrm{S}}(t)$ of the central spin can then be decomposed as $\rho_{\mathrm{S}}(t)=\sum_{m} \rho_{m}(t)$, where $\rho_{m}(t)=\operatorname{Tr}_{\mathrm{B}}\left\{\Pi_{m} \rho(t)\right\}$ with $\rho(t)$ denoting the density matrix of the total system and $\operatorname{Tr}_{B}$ the partial trace over the spin bath. Employing the correlated projection operator technique described in detail in [15-17], one derives the following system of coupled equations of motion for the dynamical variables $\rho_{m}(t)$,

$$
\begin{aligned}
\frac{\mathrm{d}}{\mathrm{d} t} \rho_{m}(t)= & \int_{0}^{t} \mathrm{~d} \tau\left\{\left[g_{m+1}(\tau)+g_{m+1}^{*}(\tau)\right] \sigma_{+} \rho_{m+1}(t) \sigma_{-}\right. \\
& +\left[f_{m-1}(\tau)+f_{m-1}^{*}(\tau)\right] \sigma_{-} \rho_{m-1}(t) \sigma_{+} \\
& -f_{m}(\tau) \sigma_{+} \sigma_{-} \rho_{m}(t)-f_{m}^{*}(\tau) \rho_{m}(t) \sigma_{+} \sigma_{-} \\
& \left.-g_{m}(\tau) \sigma_{-} \sigma_{+} \rho_{m}(t)-g_{m}^{*}(\tau) \rho_{m}(t) \sigma_{-} \sigma_{+}\right\}
\end{aligned}
$$

The quantities $f_{m}(\tau)$ and $g_{m}(\tau)$ represent certain two-point correlation functions of the spin bath and are given explicitly by [17]

$$
\begin{gathered}
f_{m}(\tau)=4 \sum_{k} \alpha_{k}^{2}\left\langle\sigma_{-}^{k} \sigma_{+}^{k} \mathrm{e}^{\mathrm{i}\left(\omega_{0}-\omega+4 K_{3}+2 \alpha_{k}\right) \tau}\right\rangle_{m}, \\
g_{m}(\tau)=4 \sum_{k} \alpha_{k}^{2}\left\langle\sigma_{+}^{k} \sigma_{-}^{k} \mathrm{e}^{\mathrm{i}\left(-\omega_{0}+\omega-4 K_{3}+2 \alpha_{k}\right) \tau}\right\rangle_{m},
\end{gathered}
$$

where $\langle\cdot\rangle_{m}$ denotes the bath average under the constraint of fixed value for the quantum number $m$.

\section{Effective Hamiltonian}

Master equation (5) clearly shows that the reduced system dynamics of the central spin is strongly influenced by the structure of the bath correlation functions defined by equations (6) and (7), depending in a complicated way on the distribution of the coupling constants $\alpha_{k}$. However, for small times $\tau$ the exponential functions in these expressions may be replaced by 1 obtaining the simpler form

$$
\begin{aligned}
f_{m}(\tau) & \simeq 4 \sum_{k} \alpha_{k}^{2}\left\langle\sigma_{-}^{k} \sigma_{+}^{k}\right\rangle_{m}=4 \frac{1}{N} \sum_{k} \alpha_{k}^{2}\left(\frac{N}{2}-m\right), \\
g_{m}(\tau) & \simeq 4 \sum_{k} \alpha_{k}^{2}\left\langle\sigma_{+}^{k} \sigma_{-}^{k}\right\rangle_{m}=4 \frac{1}{N} \sum_{k} \alpha_{k}^{2}\left(\frac{N}{2}+m\right) .
\end{aligned}
$$

The important point to note is that these approximate correlation functions coincide for small $\tau$ with the correlation functions one obtains for the model of a uniform distribution of the coupling constants that is described by the Hamiltonian

$$
H_{\text {eff }}=\frac{\omega_{0}}{2} \sigma_{3}+\sum_{k=1}^{N} \frac{\omega}{2} \sigma_{3}^{k}+\alpha_{\text {eff }} \sum_{k=1}^{N} \vec{\sigma} \cdot \vec{\sigma}^{k},
$$

where we have introduced the effective coupling constant

$$
\alpha_{\mathrm{eff}} \equiv \sqrt{\frac{1}{N} \sum_{k} \alpha_{k}^{2}}
$$

In fact, starting from the effective Hamiltonian $H_{\text {eff }}$ one obtains a second-order master equation [16] that is exactly of the structure of equation (5), where however the correlation functions take the form

$$
\begin{aligned}
& f_{m}^{\text {eff }}(\tau)=4 \alpha_{\text {eff }}^{2}\left(\frac{N}{2}-m\right) \mathrm{e}^{\mathrm{i}\left(\omega_{0}-\omega+4 \alpha_{\text {eff }} m+2 \alpha_{\text {eff }}\right) \tau}, \\
& g_{m}^{\text {eff }}(\tau)=4 \alpha_{\text {eff }}^{2}\left(\frac{N}{2}+m\right) \mathrm{e}^{\mathrm{i}\left(-\omega_{0}+\omega-4 \alpha_{\text {eff }} m+2 \alpha_{\text {eff }}\right) \tau} .
\end{aligned}
$$

Analyzing the short-time behaviour of the two-point correlation functions in the two different models, it thus appears reasonable to suppose that the short-time dynamics of the central spin is essentially unaffected by the non-uniformity of the distribution of the coupling constants. We therefore expect that the short-time behaviour of the central spin coupled to a non-uniform spin bath can be modelled through the effective Hamiltonian (10) with a single effective coupling constant that is defined by means of relationship (11). This simple prescription leads to an efficient treatment of the short-time dynamics because the uniform spin bath model with Hamiltonian (10) can easily be solved analytically (see e.g. [16]).

\section{Numerical results}

In the following we compare the dynamics corresponding to Hamiltonian (1) with the dynamics given by the effective Hamiltonian (10) with coupling constant (11). In both cases we present the exact time evolution of the central spin, using the analytical solution for the effective Hamiltonian and 

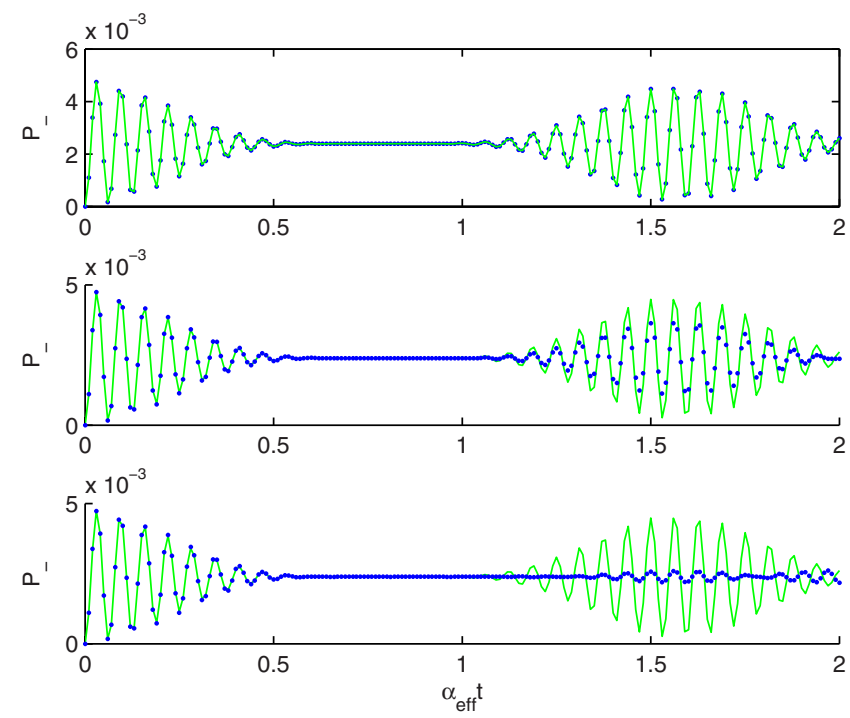

Figure 1. Population of the central spin as a function of time for Hamiltonian (1) (blue dots) and for the effective Hamiltonian (10) (green line) with $N=6$ bath spins. The couplings are given by equation (14) for a fixed $\alpha_{\text {eff }} / \omega_{0}=0.01, \omega=0$ and with $A=0$ (top), $A=0.5$ (middle) and $A=1.0$ (bottom).

a numerical simulation of the full von Neumann equation for the density matrix of the total system for the case of the non-uniform spin bath model. The initial state of the total system is assumed to be an uncorrelated product state of the form $\rho(0)=\rho_{\mathrm{S}}(0) \otimes \rho_{\mathrm{B}}(0)$. The dynamics of the central spin is represented in terms of the populations $P_{-}(t) \equiv\left\langle-\left|\rho_{\mathrm{S}}(t)\right|-\right\rangle$ and the coherences $C(t) \equiv\left\langle+\left|\rho_{\mathrm{S}}(t)\right|-\right\rangle$. The distribution of the coupling constants $\alpha_{k}$ appearing in Hamiltonian (1) is taken to be an exponential function [5]

$$
\alpha_{k}=\alpha_{0} \exp \left(-A \frac{k}{N}\right)
$$

where the constant $A$ measures the degree of the nonuniformity of the distribution. In the following, we present results for various values of $A$ keeping the corresponding effective coupling $\alpha_{\text {eff }}$ fixed.

We first consider an unpolarized, infinite-temperature initial bath state given by $\rho_{\mathrm{B}}(0)=2^{-N} I$. Figure 1 shows the behaviour of the corresponding populations $P_{-}(t)$ for three different values of the parameter $A$ and a fixed value for the effective coupling constant $\alpha_{\text {eff. }}$. We observe that for all values of the non-uniformity parameter $A$, the short-time dynamics is very well reproduced by the effective Hamiltonian, the relevant time scale being given by $t \sim \alpha_{\text {eff }}^{-1}$, as expected. For larger times and larger non-uniformity, the dynamics of $H_{\text {eff }}$ leads to partial revivals of the populations that are due to the commensurability of the system frequencies [16] and that are not present in the non-uniform model. However, we see that the initial decay of the population as well as the stationary state reached are indeed accurately described by the effective Hamiltonian.

Similar results are obtained for the coherences $C(t)$ that are shown in figure 2 . We conclude again that the decoherence behaviour of the central spin is very well predicted by the effective Hamiltonian, while for longer times an additional dephasing for stronger non-uniformity leads to the
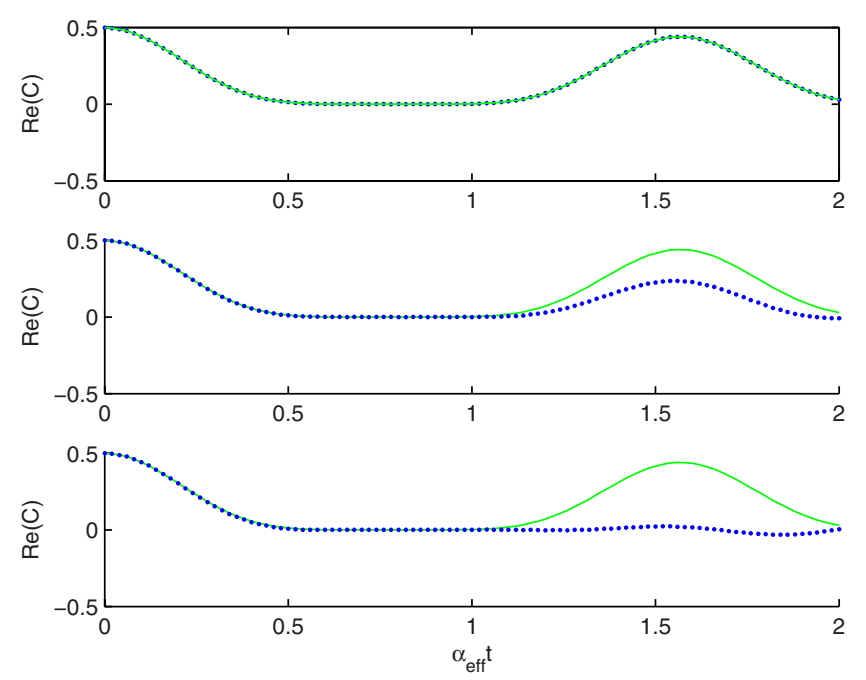

Figure 2. The same as figure 1 for the coherences.
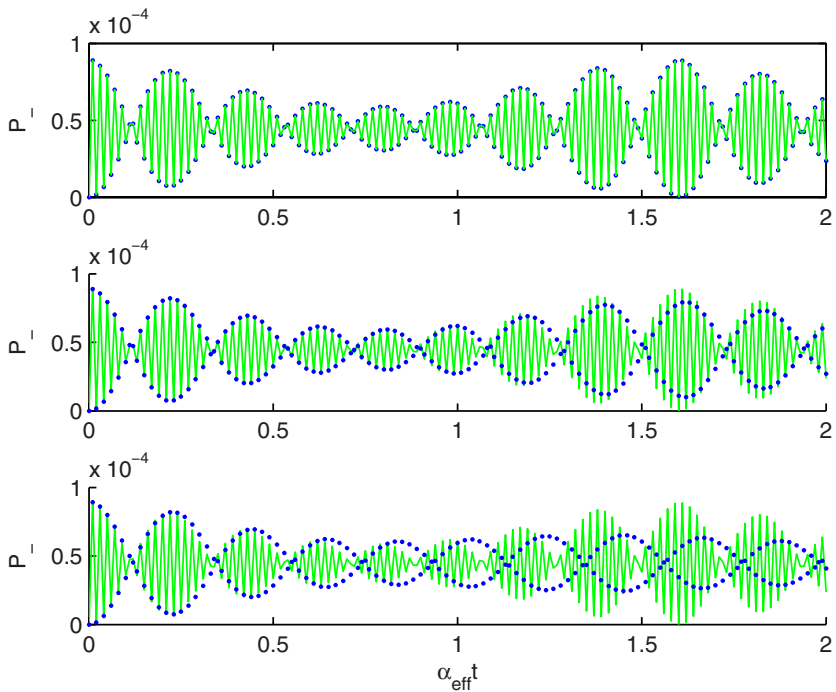

Figure 3. The same as figure 1 for the polarized initial bath state (15) with $p=0.9$ and $\omega / k T=-\ln p /(1-p)$.

disappearance of the revivals of the coherences. Analogous conclusions can be drawn for polarized (finite-temperature) initial bath states. Figure 3 shows the temporal behaviour of the populations for the case of the initial bath state

$$
\rho_{\mathrm{B}}(0)=\sum_{m} p^{N / 2+m}(1-p)^{N / 2-m} \Pi_{m}
$$

Moreover, we have checked numerically that a similar behaviour is observed if one considers various other distributions for the coupling constants $\alpha_{k}$, such as a linear or a Gaussian distribution.

\section{Conclusions}

We have developed an effective Hamiltonian approach to the non-Markovian damping and decoherence dynamics of a central spin coupled to a spin bath. An interesting extension of the present approach could be the development of a perturbation expansion that uses the dynamics given by the effective Hamiltonian as a starting point of zeroth 
order. The perturbation parameter of such an expansion would not be given by the strength of the system-bath couplings, the $\alpha_{k}$ in the example treated here, but by the degree of the non-uniformity of the distribution of the couplings, which is given in our example by quantity $A$ introduced in equation (14). The basic strategy underlying our approach could also be useful in the study of other relevant physical models featuring strong memory effects. The crucial ingredient is obviously to identify an appropriate class of effective Hamiltonian operators that is parameterized by a certain set of optimized effective variables.

\section{References}

[1] Khaetskii A V, Loss D and Glazman L 2002 Phys. Rev. Lett. 88186802

[2] Coish W A and Loss D 2004 Phys. Rev. B 70195340

[3] de Sousa R and Das Sarma S 2003 Phys. Rev. B 68115322
[4] Coish W A, Fischer J and Loss D 2008 Phys. Rev. B 77125329

[5] Deng C and Hu X 2006 Phys. Rev. B 73241303

[6] Saikin S K, Yao W and Sham L J 2007 Phys. Rev. B 75125314

[7] Loss D and Di Vincenzo D P 1998 Phys. Rev. A 57120

[8] Schliemann J, Khaetskii A and Loss D 2003 J. Phys.: Condens. Matter 15 R1809

[9] Erlingsson S I, Nazarov Y V and Falko V I 2001 Phys. Rev. B 64195306

[10] Schliemann J, Khaetskii A and Loss D 2002 Phys. Rev. B 66245303

[11] Merkulov I A, Efros A L and Rosen M 2002 Phys. Rev. B $\mathbf{6 5} 205309$

[12] Semenov Y G and Kim K W 2003 Phys. Rev. B 67073301

[13] Al-Hassanieh K A, Dobrovitski V V, Dagotto E and Harmon B N 2006 Phys. Rev. Lett. 97037204

[14] Dobrovitski V V and De Raedt H A 2003 Phys. Rev. E 67056702

[15] Breuer H-P 2007 Phys. Rev. A 75022103

[16] Fisher J and Breuer H-P 2007 Phys. Rev. A 76052119

[17] Ferraro E, Breuer H-P, Jivulescu M A, Napoli A and Messina A 2008 Phys. Rev. B 78064309 